\title{
TOPICS ON MEIXNER FAMILIES
}

\author{
MAREK BOŻEJKO \\ Instytut Matematyczny, Uniwersytet Wrockawski \\ Pl. Grunwaldzki 2/4, 50-384 Wrocław, Poland \\ E-mail: bozejko@math.uni.wroc.pl \\ NIZAR DEMNI \\ Faculty of Mathematics, Bielefeld University \\ D-33501 Bielefeld, Germany \\ E-mail:demni@math.uni-bielefeld.de
}

\begin{abstract}
We shed some light on the inter-connections between different characterizations leading to the classical Meixner family. This allows us to give free analogs of both Sheffer's and Al-Salam and Chihara's characterizations in the classical case by the use of the free derivative operator. The paper closes with a discussion of the $q$-deformed case, $|q|<1$.
\end{abstract}

1. Introduction. A remarkable and exciting feature of Mathematics is the fact that several independent and seemingly unrelated works may lead to a common result. It is often the lack of communication between various schools that prevents the full understanding of the existing inter-connections, thereby arises the problem of unifying the different approaches, which stands out as an important, useful and also tricky task. In this spirit, the so-called Meixner family of probability distributions, referred to as Meixner distributions, admits the following characterizations:

- When it first appeared in [22], the Meixner family was defined as the set of probability measures $\mu$ with finite exponential moments in a neighborhood of zero such that:

$$
\psi(z, x):=\sum_{n \geq 0} P_{n}(x) z^{n}=\frac{e^{x H(z)}}{\mathbb{E}\left(e^{X H(z)}\right)},
$$

where $H$ is analytic around $z=0$ such that $H(0)=0, H^{\prime}(0)=1, X$ is a random variable in some probability space $(\Omega, \mathscr{F}, \mathbb{P})$ with law $\mu=\mathbb{P} \circ X^{-1}$ and $\left(P_{n}\right)_{n \geq 0}$ is the set of orthogonal polynomials (OP) with respect to $\mu . \psi$ is an $\mathrm{OP}$ generating

2000 Mathematics Subject Classification: 45L54, 37A50, 60G20, 33D45.

Key words and phrases: Meixner families, orthogonal polynomials, free and quantum probability. The paper is in final form and no version of it will be published elsewhere. 
function of exponential-type. Up to translations and dilations, the Meixner family consists of Gaussian, Poisson, Gamma, negative binomial, Meixner and binomial distributions, the latter being non-infinitely-divisible in the classical sense. Another proof of this characterization was given in [18.

- Sheffer characterized $\left(P_{n}\right)_{n}$ as the set of OP of type zero $([25])$.

- Meixner laws are the solutions of a quadratic regression problem ([20]).

- Morris showed that the variance of the natural exponential family parametrized by the mean associated with a probability measure $\mu$, is an at most quadratic polynomial in the mean if and only if $\mu$ belongs to the Meixner family ([23]).

- OP with respect to Meixner laws form a distinguished subclass of the so-called Al-Salam and Chihara set of OP ([1]).

After the birth of free probability theory, for which miscellaneous analogs of known results in classical probability theory were derived, the free Meixner family was defined in [4] and covers six probability distributions called analogously to their classical counterparts free Gaussian or Wigner, free Poisson or Marchenko-Pastur, free Gamma, free negative binomial, free Meixner and free binomial, also known as the free stationary Jacobi law ([13], [15], [16]). Their study produced free versions of some of the above characterizations: the exponential-type OP generating functions are replaced by generating functions of Cauchy-Stieltjes type ([4], [10, [19]), free Meixner distributions solve a quadratic regression problem in a free setting $([9)$ and have associated Cauchy-Stieltjes type families with linear means and quadratic variances ([11]). To our best knowledge, no free analogs of Sheffer's and Al-Salam and Chihara's characterizations exist in literature.

In the present paper, we highlight some connections between the different characterizations in both the classical and free settings. More precisely, we start by explaining how Sheffer's characterization leads to Al-Salam and Chihara results and vice versa. Then we do the same for both Kubo's and Morris's characterizations. In the free setting, a connection is found between Bryc and Ismail's characterization using Cauchy-Stieljes families of quadratic variances and the one given by the authors via Cauchy-Stieltjes-type generating functions of OP $([10])$.

The study of those connections shows a parallel between the classical and free settings manifested by classical and free cumulants generating functions on the one hand and by classical and free derivatives on the other hand. As a matter of fact, it reveals what free Sheffer's and free Al-Salam and Chihara characterizations should be. Moreover, we observed that, to a given OP generating function of a probability measure satisfying suitable integrability conditions, corresponds a family of probability measures parametrized by the mean such that the variance is an at most quadratic polynomial in the mean (we will say for short 'an at most quadratic variance'). This fact is an immediate consequence of the orthogonality or equivalently the three-terms recurrence relation satisfied by the OP. Moreover, the coefficients of the variance are easily expressed in terms of the Jacobi-Szegö parameters. This general observation gives a machinery that we used to solve the problem of characterizing probability distributions $\mu_{\lambda}$ (of finite all order moments) having ultraspherical-type generating functions ([16]). The paper is closed by pointing out the difficulties arising in the $q$-deformed case, $|q|<1$. 
REMARK 1.1. The Meixner family will be referred to as the classical Meixner family. Moreover, we shall often use the word 'law' instead of 'probability distribution' and also the acronymes 'Meixner, Al-Salam Chihara, Sheffer, Kubo etc...' to refer to the corresponding characterizations cited above. We hope there will be no confusion while reading the paper.

2. On Sheffer and Al-Salam and Chihara characterizations. Few years after Meixner's classification was given, Sheffer (25]) provided an easy proof of it while studying the following general situation: let $\left(Q_{n}\right)_{n}$ be a set of polynomials, with $Q_{n}$ of degree $n$ for each $n \geq 0$, which are not necessarily orthogonal. Let $D$ denote the derivative operator and take $Q_{0}(x)=1$. Then there exists a (unique) differential operator $J:=\sum_{n \geq 1} c_{n} D^{n}$ with constant coefficients $\left(c_{n}\right)_{n}, c_{1} \neq 0$ such that $J Q_{n}=Q_{n-1}, Q_{-1}=0 .\left(Q_{n}\right)_{n}$ is then said to be a set of type zero with respect to $J$ or belongs to $J$. Sheffer showed that being a set of type zero is equivalent to

$$
\sum_{n \geq 0} Q_{n}(x) z^{n}=A(z) e^{x H(z)}
$$

where $A$ is an entire function around zero (called the determining function) and $H$ is the inverse function of $J$ when the latter is viewed as an entire function (of course, the series defining $J$ is supposed to be a convergent). It is then obvious that one immediately recovers the Meixner family under the additional orthogonality assumption on $\left(Q_{n}\right)_{n}$. In the middle 70's, Al-Salam and Chihara ([1]) adressed the following problem: characterize the set of OP $\left(r_{n}\right)_{n},\left(q_{n}\right)_{n}$ such that their convolution

$$
s_{n}(x, y)=\sum_{k=0}^{n} r_{k}(x) q_{n-k}(y)
$$

defines a set of OP in the variable $x$ for all $y \cdot\left(r_{n}\right)_{n}$ and $\left(q_{n}\right)_{n}$ belong then to the so-called Al-Salam and Chihara set of OP. If one further requires that $s_{n}(x, y):=p_{n}(x+y)$ for some polynomial $p_{n}$, then $r_{n}=q_{n}$ for all $n$ and $\left(r_{n}\right)_{n}$ are OP with respect to a probability measure from the classical Meixner family.

REMARK 2.1. This last characterization was first noticed by Lancaster in [21] and we would like to thank Prof. Gérard Letac for helping us with this reference.

2.1. From Sheffer to Al-Salam and Chihara. Let us now see how one carries over the Al-Salam and Chihara problem into the setting of Sheffer. Let $\left(r_{n}\right)_{n},\left(q_{n}\right)_{n},\left(p_{n}\right)_{n}$ be three sets of OP related by the convolution

$$
p_{n}(x+y)=\sum_{k=0}^{n} r_{k}(x) q_{n-k}(y)
$$

and let us prove that $\left(r_{n}\right),\left(q_{n}\right)$ are of type zero with respect to the same operator. Denote by $J$ and $\tilde{J}$ the (unique) operators ([25]) such that $J\left(r_{n}\right)=r_{n-1}$ and $\tilde{J}\left(q_{n}\right)=q_{n-1}$ and write

$$
J=\sum_{k \geq 1} c_{k}(x) D^{k}, \quad \tilde{J}=\sum_{k \geq 1} \tilde{c}_{k}(x) D^{k}
$$


where $c_{k}, \tilde{c}_{k}$ are polynomials of degree at most $k-1$ and $c_{1}=\tilde{c}_{1}=1$. Note first that if (4) holds, then $c_{n}(x)=\tilde{c}_{n}(x)$ for all $n$ and this follows from the symmetry of $(x, y) \mapsto$ $p_{n}(x+y)$ in both arguments. More precisely,

$$
\begin{aligned}
J\left(p_{n}(\cdot+y)\right)(x) & =\sum_{k=1}^{n} r_{k-1}(x) q_{n-k}(y)=p_{n-1}(x+y) \\
& =\sum_{k=0}^{n-1} r_{k}(y) q_{n-k-1}(x)=\tilde{J}\left(p_{n}(\cdot+y)\right)(x),
\end{aligned}
$$

which proves our claim by induction on the degree $n$. Again, the symmetry gives

$$
p_{n-1}(x+y)=J\left(p_{n}(\cdot+y)\right)(x)=J\left(p_{n}(x+\cdot)\right)(y),
$$

which yields $c_{n}(x)=c_{n}(y)=c_{n}$ for all $n$. As a result, $\left(r_{n}\right)_{n},\left(q_{n}\right)_{n}$ are of type zero with respect to the same operator $J$ (hence the same $H$ ) and Sheffer's result applies.

REMARK 2.2. Since the expectation of $\psi(z, X)$ equals 1 when $\left(P_{n}\right)_{n}$ is a set of OP, then $A(z)=1 / \mathbb{E}\left(e^{H(z) x}\right)$ and is then entirely determined by the knowledge of $H$. Thus, $r_{n}=q_{n}$ for all $n$ whenever both sets of OP belong to the same lowering operator $J$. This claim is no more true for sets of not necessarily OP.

2.2. From Al-Salam and Chihara to Sheffer. Consider a set $\left(r_{n}\right)_{n}$ of OP with respect to a probability distribution $\mu$ having a finite exponential moment in a neighborhood of zero and assume that it is of type zero with respect to $J$ :

$$
J=\sum_{n \geq 1} c_{n} D^{n}, c_{1}=1
$$

Form the convolution

$$
s_{n}(x, y)=\sum_{k=0}^{n} r_{k}(x) r_{n-k}(y),
$$

it then follows that $\left.J s_{n}(\cdot, y)\right)=J\left(s_{n}(x, \cdot)\right)$, which implies (by induction) that $s_{n}(x, y)=$ $p_{n}(x+y)$ for a certain polynomial $p_{n}$. The orthogonality of $\left(p_{n}(\cdot+y)\right)_{n}$ follows from

$$
\begin{array}{r}
\int_{\mathbb{R}} p_{n}(x+t) p_{m}(x+t) \mu(d x) \mu(d t)=\sum_{k=0}^{n} \sum_{l=0}^{m} \int_{\mathbb{R}} r_{k}(x) r_{l}(x) \mu(d x) \int_{\mathbb{R}} r_{n-k}(t) r_{m-l}(t) \mu(d t) \\
=\sum_{k=0}^{n} \sum_{l=0}^{m}\left\|r_{k}\right\|^{2}\left\|r_{n-k}\right\|^{2} \delta_{k l} \delta_{n-k, m-l}=\sum_{k=0}^{n}\left\|r_{k}\right\|^{2}\left\|r_{n-k}\right\|^{2} \delta_{n, m},
\end{array}
$$

where $\mu$ is the orthogonality probability distribution of $\left(r_{n}\right)_{n}$.

REMARK 2.3. Since

$$
p_{n}(x)=\sum_{k=0}^{n} r_{k}(x-y) q_{n-k}(y),
$$

for all $y,\left(p_{n}\right)_{n}$ belongs to $J$ too and by orthogonality, $p_{n}=r_{n}$ for all $n$.

3. On Kubo and Morris characterizations. In the early eighties, the classical Meixner family appeared in [23] as the set of probability measures which generate natural 
exponential families parametrized by the means, such that the variance of a given exponential family is at most quadratic in the mean. More precisely, given a (non-degenerate) probability measure $\mu$ with finite exponential moments in some neighborhood of zero, its natural exponential family is the one-parameter family of probability measures defined by

$$
\mathbb{P}_{\theta}(d x):=\frac{e^{\theta x}}{\mathbb{E}\left(e^{\theta X}\right)} \mu(d x) .
$$

Let $z$ and $V$ denote respectively the mean and the variance of $\mathbb{P}_{\theta}$ for a given $\theta$. Then $z=\tau^{\prime}(\theta)$ and $V=\tau^{\prime \prime}(\theta)$ where

$$
\tau(\theta):=\log \mathbb{E}\left(e^{\theta X}\right)
$$

is the cumulants generating function of $\mu$. Since $V=V(\theta)>0$, then $\tau^{\prime}$ is invertible and $\theta=\left(\tau^{\prime}\right)^{-1}(z):=S(z)$ makes sense for small $z$. We also have $S(0)=0, S^{\prime}(z)=1 / V(z)$ and $S^{\prime}(0) \neq 0$ which one may suppose to equal 1 . This gives rise to a new exponential family parametrized by the mean $z$

$$
\mathbb{P}_{z}(d x):=\frac{e^{S(z) x}}{\mathbb{E}\left(e^{S(z) X}\right)} \mu(d x)
$$

of mean $z$ and variance $V(z)=\tau^{\prime \prime}(S(z))$. It was shown in 23] that $V$ is at most quadratic in $z$ if and only if $\mu$ belongs to the classical Meixner family.

Few years ago, another proof of Meixner's classification was given in ([18]) via OP generating functions using Asai-Kuo-Kubo's criterion ([2]). Keeping the notations in (2), the author proved that (2) generates orthogonal polynomials if and only if

$$
b H^{-1}(z)+a=\tau^{\prime}(z),
$$

where $a \in \mathbb{R}, b>0$ are respectively the mean and the variance of the orthogonality measure and

$$
H^{\prime}(z)=\frac{1}{\beta z^{2}+\gamma z+1}, \quad(\beta \geq 0) \text { or }(\beta<0, b / \beta \in \mathbb{R} \backslash \mathbb{Z}) .
$$

The values $(\beta<0, b / \beta \in \mathbb{Z})$ correspond to a signed measure. Note that, for $a=0, b=1$. (5) together with (6) give a Riccati-type equation for $\tau^{\prime}$ :

$$
\tau^{\prime \prime}(z)=\left(H^{-1}\right)^{\prime}(z)=\frac{1}{H^{\prime}\left(\tau^{\prime}(z)\right)}=\beta\left(\tau^{\prime}\right)^{2}(z)+\gamma \tau^{\prime}(z)+1
$$

3.1. From Kubo to Morris. It is known that the analyticity assumptions on $S$ (since $S^{-1}=\tau^{\prime}$ and $\tau^{\prime}$ is analytic near $z=0$ ) allow one to expand

$$
\frac{e^{S(z) x}}{\mathbb{E}\left(e^{S(z) x}\right)}=\sum_{n \geq 0} P_{n}(x) z^{n},
$$

where $P_{n}$ is a polynomial of exact degree $n$ and $\left(P_{n}\right)_{n \geq 0}$ need not to be orthogonal (see [24] p. 45). Assume that $\mu$ has zero mean and unit variance (standard), then one uses Kubo's results above after identifying $S$ with $H$ so that $S^{\prime}(z)=H^{\prime}(z)=1 / V(z)$ fits (6) (one further needs to check the sign of the parameters involved in the variance). 
3.2. From Morris to Kubo. Let $\left(V_{n}\right)_{n}$ be the set of monic OP with respect to the standard $\mu$ and consider the exponential generating function

$$
\frac{e^{H(z) x}}{\mathbb{E}\left(e^{H(z) X}\right)}=\sum_{n \geq 0} a_{n} V_{n}(x) z^{n}=\sum_{n \geq 0} \frac{1}{n !} V_{n}(x) z^{n}
$$

where the latter equality follows from substituting $x$ with $x / z$ then letting $z \rightarrow 0$ and finally using $H^{\prime}(0)=1$. It is known that

$$
x V_{n}(x)=V_{n+1}(x)+\alpha_{n} V_{n}(x)+\omega_{n} V_{n-1}(x), \quad V_{-1}:=0, \omega_{0}:=1
$$

where $\left(\alpha_{n}\right)_{n},\left(\omega_{n}\right)_{n}$ are the Jacobi-Szegö parameters $([14])$. Then, inverting the order of integration and using the orthogonality of $\left(V_{n}\right)_{n}$, it easily follows that

$$
m(z):=\sum_{n \geq 0} \int_{\mathbb{R}} x V_{n}(x) \mu(d x) \frac{z^{n}}{n !}=\alpha_{0}+\omega_{1} z=z
$$

since $\mu$ is standard. Similarly, one gets

$$
V(z)=\left(\omega_{2}-2\right) \frac{z^{2}}{2}+\alpha_{1} z+1
$$

which fits into the work of Morris and identifies the coefficients $\beta, \gamma$ in $(6)$.

What is quite interesting is that every OP generating function of a given measure satisfying suitable integrabiltiy conditions defines a one-parameter family of probability measures parametrized by the mean such that, for a fixed value of the parameter (the mean), the variance is at most quadratic in the mean. When the OP generating function is manageable (which is not the case for instance for the $q$-Meixner family due to the infinite product, [5]), one gets a machinery that can be used to characterize a family of probability measures via their OP generating functions. This is, as we will see, the case of the free Meixner family with Cauchy-Stieltjes-type OP generating functions and of a natural generalization of ultraspherical polynomials ([16]).

4. Free Meixner family. A parallel definition of Lévy-Meixner processes in free probability theory appeared in [4] after the pioneering work of Biane [7] on free processes with free increments, where the author derived free analogs of exponential martingales involving free cumulant generating functions. Then, the free Meixner family was defined in [9] by proving a free Laha-Lukacs characterization and it consists of six laws which we think of as the free analogs of the six laws forming the classical Meixner family (see the introduction). Only the free binomial distribution is not infinitely divisible with respect to the free additive convolution. Similarly to the classical case, one can find the connection between Bryc and Ismail characterization via Cauchy-Stieltjes families with at most quadratic variances and the one recently given by the authors via Cauchy-Stieltjes-type OP generating functions ([9]).

On the one hand, a combination of [4] and 9] allowed us to characterize the free Meixner distributions and Asai-Kuo-Kubo criterion ([2]) was used to derive analogous identities to (5) and (6) involving the free cumulant generating function ([10]). More precisely, let $\mu$ be a standard probability distribution with all moments finite. Then, $\mu$ 
belongs to the free Meixner family if and only if

$$
\psi(z, x):=\sum_{n \geq 0} P_{n}(x) z^{n}=\frac{1}{u(z)[f(z)-x]}
$$

where

$$
\begin{aligned}
f(z)=K(u(z)) & =z+\frac{1}{u(z)}, \\
\frac{u(z)}{z} & =\frac{1}{1+a z+b z^{2}},
\end{aligned}
$$

for $a \in \mathbb{R}, b \geq-1$ (parameters of $\mu$, not to be confused with $a, b$ used previously), where $K$ is the right inverse in a neighborhood of zero of the Cauchy-Stieltjes transform of $\mu$ :

$$
G(z):=\int \frac{1}{z-x} \mu(d x), \quad z \in \mathbb{C} \backslash \operatorname{supp}(\mu) .
$$

The second equality in 10 is analogous to (5) if it is written as $R(u(z))=z$ where $R(z)=K(z)-1 / z$ is the free cumulant generating function, while [11) is analogous to (6) where the classical derivative is replaced by the free derivative defined by

$$
\left\{\begin{array}{l}
D^{0}(f)(z)=\frac{f(z)-f(0)}{z}, \quad z \neq 0, \\
D^{0}(f)(0)=f^{\prime}(0) .
\end{array}\right.
$$

On the other hand, to a compactly-supported probability measure is associated a Cauchy-Stieltjes family defined by ([12])

$$
\left\{\mathbb{P}_{\theta}(d x):=\frac{1}{M(\theta)} \frac{1}{1-\theta x} \mu(d x)\right\},
$$

for small complex $\theta$, where $M$ is the moment generating function defined by

$$
M(\theta):=\int_{\mathbb{R}} \frac{1}{1-\theta x} \mu(d x)=\frac{1}{\theta} G\left(\frac{1}{\theta}\right) .
$$

If $\mu$ is not a Dirac mass, then the mean of $\mathbb{P}_{\theta}$

$$
z:=\int_{\mathbb{R}} x \mathbb{P}_{\theta}(d x)=\frac{M(\theta)-1}{\theta M(\theta)}:=k(\theta)
$$

is invertible as a function of small enough $\theta$, thus a new parametrization by the mean is performed on $\mathbb{P}_{\theta}$ giving rise to

$$
\mathbb{P}_{z}(d x):=\frac{1}{M(v(z))} \frac{1}{1-v(z) x} \mu(d x), \quad \theta:=k^{-1}(z)=v(z) .
$$

Let $V=V(z)$ denote the variance of $P_{z}$ for $z$ small enough. Then

$$
\mathbb{P}_{z}(d x):=\frac{V(z)}{V(z)+z(x-z)} \mu(d x)
$$

when $\mu$ has mean zero. It was shown in [12] that $V$ is quadratic if and only if $\mu$ belongs to the free Meixner family. To deduce this result using OP generating functions, expand the ratio in 13 as

$$
\frac{V(z)}{V(z)+z(x-z)}=\sum_{n \geq 0} P_{n}(x) z^{n}
$$


with not necessarily OP. Set $u(z):=z / V(z)$ with $V(z)=1+a z+b z^{2}$. Then equation (2.1) in [12 fits the second equality in 10 . It follows that the density in 13 is given by (9) which gives the result.

Conversely, suppose we are given a standard probability measure with all moments finite and the $\mathrm{OP}$ generating function $(9)$ with $u(0)=0, u^{\prime}(0)=1, u f(0)=1$. Then, one defines a Cauchy-Stieltjes family $\left(\mathbb{P}_{z}\right)_{z}$ parametrized by the mean with an at most quadratic variance. Since $z \mapsto R(z)$ is defined and analytic in a neighborhood of zero, $\mu$ is compactly-supported ([6]). Moreover, setting $V(z)=z / u(z), \mathbb{P}_{z}$ takes the form of (13) so that Theorem 1.2 in [12] applies.

5. Free-type zero polynomials and free Sheffer characterization. Let $\left(U_{n}\right)_{n}$ be the set of monic Tchebycheff polynomials of the second kind ([14]) defined by

$$
\sum_{n \geq 0} U_{n}(x) z^{n}=\frac{1}{1-z x+z^{2}} .
$$

It is easy to see from the above OP generating function that $\left(U_{n}\right)_{n}$ can not be a set of finite $A, B, C$-types described in [25] (the $A$-type includes the type zero). This was already noticed for the Legendre polynomials (see p. 621-622 in [25]) and the same reasoning applies for $\left(U_{n}\right)_{n}$. This is due to the fact that the above generating function is not of an exponential type (A-type) and its derivative with respect to either $x$ or $z$ will raise it to the square so that, for each $n$, the polynomial coefficients in the expansion of $U_{n}^{\prime}$ (B-type) and $n U_{n}$ (C-type) in terms of $\left(U_{n-1}, \ldots, U_{0}\right)$ have unbounded degrees in $n$. Nevertheless, they will be of type zero in the free sense, that is, when substituting the classical derivative $D$ by its free analog $D^{0}$ defined by 12 and the exponential function by $x \mapsto(1-x)^{-1}$. Following Sheffer, we first define 'free differential operators' by

$$
J^{0}:=\sum_{n \geq 0} c_{n}(x)\left(D^{0}\right)^{n}
$$

where $c_{n}$ are polynomials. We will say that a set of polynomials $\left(P_{n}\right)_{n}$ (where $P_{n}$ has degree $n$ ) belongs to $J$ if $J^{0} P_{n}=P_{n-1}, P_{0} \neq 0, P_{-1}:=0$. Since $\left(D^{0}\right)^{k} x^{n}=x^{n-k}$ for $1 \leq k \leq n$ and $D^{0} 1=0$, then the sum defining $J^{0}$ terminates when acting on polynomials. In addition, $J^{0}$ maps $x^{n}$ to a polynomial of degree $\leq n-1$ if and only if $c_{0}=0$ and the degree of $c_{k}, 1 \leq k \leq n$ does not exceed $k-1$. Writing in that case

$$
c_{k}(x)=c_{k, 0}+c_{k, 1} x+\cdots+c_{k, k-1} x^{k-1}, \quad 1 \leq k \leq n,
$$

$J^{0}$ maps $x^{n}$ to a polynomial of exact degree $n-1$ if and only if there exist at least $k$ such that $c_{k, k-1} \neq 0$.

Second, to a given set of polynomials $\left(P_{n}\right)_{n}$ corresponds a unique free differential operator $J^{0}$ such that $J^{0} P_{n}=P_{n-1}$ and $c_{1} \neq 0$, for otherwise $P_{1}$ will be constant which disagrees the fact that $\left(P_{n}\right)_{n}$ is a set of polynomials. Recall also $([25])$ that $\left(P_{n}\right)_{n}$ and $\left(Q_{n}\right)_{n}$ belong to the same operator $J^{0}$ if and only if there exists a sequence $\left(a_{n}\right)_{n \geq 0}$ such that for each $n$

$$
P_{n}(x)=a_{0} Q_{n}(x)+\cdots+a_{n-1} Q_{1}(x)+a_{n} Q_{0}(x), a_{0} \neq 0 .
$$


Finally, $\left(P_{n}\right)_{n}$ is said to be of free type $k, k \in \mathbb{N}$ with respect to $J^{0}$ if it belongs to $J^{0}$ and, for all $n \geq 1$, the degree of $c_{n}$ is at most $k$. Thus, a set of free type zero if and only if $c_{n}(x)=c_{n} \in \mathbb{R}$ for all $n \geq 1$.

Let us suppose that $c_{1}=1$ (which holds for instance if $\left(P_{n}\right)_{n}$ are monic) such that $J^{0}$, viewed as an entire series, is invertible near 0 with inverse $\rho$ with $\rho^{\prime}(0)=1$. Since $D^{0}$ acts on $x \mapsto(1-z x)^{-1}$ by multiplication by $z$ similarly as $D$ does on $x \mapsto e^{z x}$, we claim that a set of polynomials $\left(P_{n}\right)_{n}$ is of free type zero if and only if

$$
\sum_{n \geq 0} P_{n}(x) z^{n}=A(z)(1-\rho(z) x)^{-1}, \quad A(0)=\sum_{n \geq 0} a_{n} z_{n} .
$$

As a result, the free Sheffer characterization is stated as: $\left(P_{n}\right)_{n}$ is a set of monic OP with respect to a probability measure $\mu$ with finite all order moments and of free-type zero with respect to a given $J^{0}$ if and only $\mu$ belongs to the free Meixner family with $\rho(J(z))=z$. Recall from 10 that:

$$
\rho(z)=\frac{1}{f(z)}=\frac{1}{K(u(z))}, \quad A(z)=\frac{1}{f(z) u(z)}=\frac{f(z)-z}{f(z)}=1-z \rho(z)
$$

when $\mu$ is a standard free Meixner distribution of parameters $a \in \mathbb{R}, b \geq-1$. This shows that the knowledge of $J$ is enough to characterize $\left(P_{n}\right)_{n}$ and implies that $J(z)=$ $R(G(1 / z)$ ) for small enough $z$ (recall that $R(u(z))=z$ ). Using the relation

$$
R(G(z))+\frac{1}{G(z)}=z
$$

it follows that

$$
J(z)=\frac{1}{z}-\frac{1}{G(1 / z)}=\frac{1}{z}-F\left(\frac{1}{z}\right) .
$$

REMARK 5.1. The lowering operator $J$ was used in [4] when characterizing OP of CauchyStieltjes type generating functions. However, no explicit expression as a free differential operator with constant coefficients was given.

6. Free Al-Salam and Chihara characterization. Let $\mu$ be a standard free Meixner distribution with parameters $a \in \mathbb{R}, b \geq-1$ and $\left(P_{n}\right)_{n}$ be the set of monic OP with respect to $\mu$. Recall that these polynomials satisfy the three-terms recurrence relation $([4])$

$$
x P_{n}(x)=P_{n+1}(x)+a P_{n}(x)+(1+b) P_{n-1}(x), \quad n \geq 2
$$

with $P_{0}(x)=1, P_{1}(x)=x-a$, and that

$$
P_{n}(x)=(1+b)^{n / 2} U_{n}(x-a, \sqrt{1+b})+a U_{n-1}(x-a, \sqrt{1+b})+b U_{n-2}(x-a, \sqrt{1+b})
$$

where

$$
U_{n}(x-a, \sqrt{1+b}):=(1+b)^{n / 2} U_{n}\left(\frac{x-a}{\sqrt{1+b}}\right)
$$

is the monic shifted Tchebycheff polynomial of the second kind. Using the elementary relation

$$
\frac{x-y}{(z-x)(z-y)}=\frac{1}{z-x}-\frac{1}{z-y}, \quad x \neq y
$$


we have

$$
\sum_{n \geq 0} \frac{P_{n}(x)-P_{n}(y)}{x-y} z^{n}=u(z) \psi(z, x) \psi(z, y)
$$

where $\psi$ is given by $\left(9\right.$. Since and $P_{0}(x)=P_{0}(y)=1$ and $z / u(z)=1+a z+b z^{2}$, we obtain

$$
\left(1+a z+b z^{2}\right) \sum_{n \geq 0} \frac{P_{n+1}(x)-P_{n+1}(y)}{x-y} z^{n}=\psi(z, x) \psi(z, y) .
$$

Set

$$
R_{n}(x, y):=\frac{P_{n+1}(x)-P_{n+1}(y)}{x-y} .
$$

Then $R_{0}(x)=1$ and $R_{n}$ is a linear combination of

$$
\frac{x^{k+1}-y^{k+1}}{x-y}=x^{k}+x^{k-1} y+\cdots+x y^{k-1}+y^{k}=\sum_{r=0}^{k} y^{r}\left(D_{x}^{0}\right)^{r}\left(x^{k}\right)=\sum_{r=0}^{k} x^{r}\left(D_{y}^{0}\right)^{r}\left(y^{k}\right)
$$

as one easily checks. Thus, (7) is equivalent to

$$
\sum_{k=0}^{n} P_{k}(x) P_{n-k}(y)=R_{n}(x, y)+a R_{n-1}(x, y)+b R_{n-2}(x, y), R_{-1}=R_{-2}:=0 .
$$

For $a=b=0$, one has

$$
\sum_{k=0}^{n} U_{k}(x) U_{n-k}(y)=\frac{U_{n+1}(x)-U_{n+1}(y)}{x-y}
$$

which was already considered in [5] when studying free Appell sets. One also easily checks that if a polynomial $s_{n}$ in two variables $(x, y)$ of total degree $n$ satisfies the Cauchy equation $D_{x}^{0} s_{n}=D_{y}^{0} s_{n}$, then it is a linear combination of $x^{k}+x^{k-1} y+x y^{k-1}+y^{k}, 0 \leq$ $k \leq n$. Therefore, the free Al-Salam and Chihara characterization is stated as

Proposition 6.1. Let $\left(r_{n}\right)_{n},\left(q_{n}\right)_{n}$ be two sets of monic OP and let

$$
s_{n}(x, y)=\sum_{k=0}^{n} r_{n}(x) q_{n-k}(y)
$$

be their convolution. Then, $s_{n}$ has the expansion

$$
s_{n}(x, y)=\sum_{k=0}^{n} b_{k} \frac{x^{k+1}-y^{k+1}}{x-y}
$$

if and only if $r_{n}=q_{n}$ for all $n$ and $\left(r_{n}\right)_{n}$ belongs to the free Meixner family.

REMARKS. 1. A remarkable difference with the classical setting is the fact that $\left(s_{n}(\cdot, y)\right)_{n}$ no longer defines a set of OP. Let us for instance consider the case of monic Tchebycheff polynomials $\left(U_{n}\right)_{n}$. Then one easily derives for all $n \geq 1$,

$$
x R_{n}(x, y)=R_{n+1}(x, y)+R_{n-1}(x, y)-U_{n+1}(y) .
$$

This can also be noticed from Al-Salam and Chihara's paper [1], where it is shown that, in order to get the desired orthogonality, it is not allowed, except for the classical Meixner family, to take $r_{n}=q_{n}, n \geq 1$. 
2. Integrating 18 with respect to $\mu$, one obtains

$$
Q_{n}(x)+a Q_{n-1}(x)+b Q_{n-2}(x)=P_{n}(x), \quad Q_{n}(x):=\int_{\mathbb{R}} \frac{P_{n+1}(x)-P_{n+1}(y)}{x-y} \mu(d y) .
$$

$Q_{n-1}$ is the $n$-th numerator polynomial of $P_{n}$ ([14]) and $Q_{n}$ is known to be equal to $U_{n}(x-a, \sqrt{1+b})([14]$ p. 89). Thus, the above equality coincides with 16$)$.

Proof of Proposition 6.1. The sufficiency was already proved and the necessity can be proved using the free Sheffer characterization. Indeed, let

$$
J=\sum_{k \geq 1} c_{k}(x)\left(D^{0}\right)^{k}, \quad \tilde{J}=\sum_{k \geq 1} \tilde{c}_{k}(x)\left(D^{0}\right)^{k},
$$

denote the unique operators satisfying $J\left(r_{n}\right)=r_{n-1}, \tilde{J}\left(q_{n}\right)=q_{n-1}\left(c_{1}=\tilde{c}_{1}=1\right)$. Then, by symmetry of $(x, y) \mapsto s_{n}(x, y)$ in both variables, we first get that $J=\tilde{J}$. Using

$$
\begin{aligned}
D_{x}^{0}\left(x^{k}+x^{k-1} y+\cdots+x y^{k-1}+y^{k}\right) & =x^{k-1}+x^{k-2} y+\cdots+x y^{k-2}+y^{k-1} \\
& =D_{y}^{0}\left(x^{k}+x^{k-1} y+\cdots+x y^{k-1}+y^{k}\right)
\end{aligned}
$$

for $k \geq 1$, one has $J^{0} s_{n}(\cdot, y)=J^{0} s_{n}(x, \cdot)$. Thus, $c_{n}(x)=c_{n}(y)=c_{n}, n \geq 1$ by induction on $n$, for some constants $\left(c_{n}\right)_{n}$ so that $\left(r_{n}\right)_{n}$ is a family of OP of type zero. Moreover, we have already seen that a set of OP is entirely determined by the knowledge of $J$ thereby $r_{n}=q_{n}$.

6.1. Wick product. We have already seen that for the classical Meixner family, the convolution polynomial $s_{n}(x, y)=p_{n}(x+y)$ for some polynomial $p_{n}$ in one variable. In the free setting, we have a similar relation but with noncommutative variables and even more, it is related to the so-called Wick product ([8]).

Let $(\mathscr{H},\langle\cdot\rangle)$ be a complex Hilbert space and let $\mathscr{H}^{\otimes n}$ be the $n$-fold tensor product of $H$ with $\mathscr{H}^{\otimes 0}=\mathbb{C} \Omega$ for a distinguished unit-norm vector $\Omega \in \mathscr{H}$ called the vacuum. Let $\Gamma_{0}(\mathscr{H})$ denote the full Fock space associated with $H$ defined by

$$
\Gamma_{0}(\mathscr{H}):=\bigoplus_{n \geq 0} \mathscr{H}^{\otimes n}
$$

and for $f \in \mathscr{H}$, consider the creation and the annihilation operators on $\Gamma_{0}(\mathscr{H})$ defined respectively by

$$
\begin{aligned}
a_{f} \Omega & =f \\
a_{f}\left(f_{1} \otimes f_{2} \otimes \cdots \otimes f_{n}\right) & =\left\langle f, f_{1}\right\rangle f_{2} \otimes \cdots \otimes f_{n}
\end{aligned}
$$

and

$$
\begin{aligned}
a_{f}^{\star} \Omega & =0, \\
a_{f}^{\star}\left(f_{1} \otimes f_{2} \cdots \otimes f_{n}\right) & =f \otimes f_{1} \otimes f_{2} \otimes \cdots \otimes f_{n} .
\end{aligned}
$$

These operators satisfy the free commutation relation

$$
a_{f}^{\star} a_{f}=\langle f, f\rangle \mathbf{1},
$$

where $\mathbf{1}$ is the identity operator $([[])$. Take $f \in \mathscr{H}$ such that $\langle f, f\rangle=1$. Then it was shown in [8] that for all $n$

$$
\left(a_{f}^{\star}\right)^{n}+\left(a_{f}^{\star}\right)^{n-1} a_{f}+\cdots+a_{f}^{\star} a_{f}^{n-1}+a_{f}^{n}=U_{n}\left(a_{f}+a_{f}^{\star}\right)
$$


and it is known as the free Wick product. As a result, with the same notations of the above Proposition,

$$
s_{n}\left(a_{f}, a_{f}^{\star}\right)=\sum_{k=0}^{n} b_{k} U_{k}\left(a_{f}+a_{f}^{\star}\right) .
$$

7. Concluding remarks: the $q$-deformed Meixner family, $|q|<1$. Though both the classical and free settings provide different characterizations of their corresponding Meixner families, the $q$-deformed setting is rather complicated and we record below the main difficulties arising in this case. Let us first recall that the $q$-deformed Meixner family was already defined in [5] and its elements are (up to a rescaling) the orthogonality measures of the Al-Salam and Chihara OP characterized by the following property: $\left(r_{n}\right)_{n}$ and $\left(q_{n}\right)_{n}$ belong to the Al-Salam and Chihara family of OP if and only if their convolution

$$
s_{n}(x, y)=\sum_{k=0}^{n} r_{n}(x) q_{n-k}(y)
$$

defines a set of $\mathrm{OP}$ in $x$ for infinitely many $y([1])$. Up to a rescaling, their generating function is given by ([1], p. 14)

$$
\psi_{q}(z, x)=\prod_{k=0}^{\infty} \frac{1+a q^{k} z+b q^{2 k} z^{2}}{1+(a-(1-q) x) q^{k} z+(b+1-q) q^{2 k} z^{2}},
$$

for $a \in \mathbb{R}, b \geq-1+\max (q, 0)$, which may be written as

$$
\psi_{q}(z, x)=A_{q}(z) \prod_{k=0}^{\infty} \frac{1}{1-(1-q) q^{k} H_{k}(q, z) x}
$$

for some analytic function $H(q, \cdot)$ around zero with $H(q, 0)=0, H^{\prime}(q, 0) \neq 0$. Thus, $\psi_{q}$ can not in general be expressed through the $q$-exponential function $e_{q}$ defined by

$$
e_{q}(x):=\sum_{k \geq 0} \frac{x^{k}}{[k]_{q} !}=\prod_{k=0}^{\infty} \frac{1}{1-(1-q) q^{k} x}
$$

whenever it makes sense. The difficulties arising in this case sum up into the infinite products or the infinite sums involved in the expressions, which reduce to only one term when $q=0$ :

1. The infinite product form of $\psi_{q}$ prevents one from deriving $q$-analogs of relations (5), (6), (10), (11) by performing direct computations of the first and the second moment.

2. The following characterization via $q$-exponential families was given in [11]: a $q$ exponential family associated with a compactly-supported measure and parametrized by the mean has an at most quadratic variance if and only if the measure belongs to the $q$-Meixner family. Thus, regarding relations $(6)$ and (11), one considers a quadratic variance function

$$
V(z)=b z^{2}+a z+1, \quad b \geq-1+\max (q, 0), a \in \mathbb{R}
$$

of a $q$-Meixner distribution and defines $f$ by

$$
D^{q} f(q, z)=\frac{1}{V(z)}, \quad f(q, 0)=0, z \in V(0),
$$

where $D^{q}$ is the $q$-derivative defined by 
It is easy to see that

$$
D^{q} f(q, z):=\frac{f(q, z)-f(q, q z)}{(1-q) z} .
$$

$$
f(q, z)=(1-q) \sum_{k \geq 0} \frac{q^{k} z}{1+a q^{k} z+b q^{2 k} z^{2}} .
$$

Then, $f$ is invertible and we think of its inverse as a $q$-analog of the free cumulant generating function for $|q|<1$. Unfortunately, the inversion procedure is a hard task (use Lagrange's formula for instance).

3. Though $e_{q}$ is uniquely determined by the requirement

$$
D_{x}^{q}\left(e_{q}(z x)\right)=z e_{q}(z x),
$$

it is not immediate that $\psi_{q}$ is given by 19 since the function $H$ may depend on both $k$ and $q$.

4. Finally, the dependence of $H$ on $q$ shows that

$$
D_{q}^{x}\left[\psi_{q}(z, x) \psi_{q}(z, y)\right] \neq D_{q}^{x}\left[\psi_{q}(z, x) \psi_{q}(z, y)\right]
$$

in general (except in the case $a=b=0$ corresponding to the $q$-Hermite polynomials, [8]). Nevertheless, a polynomial $s_{n}$ in two variables $x, y$ of degree $n$ satisfies the Cauchy equation $D_{x}^{q} s_{n}=D_{y}^{q} s_{n}$ if and only if $s_{n}$ is a linear combination of the homogeneous polynomials

$$
h_{k}(x, y):=\sum_{k=0}^{n}\left(\begin{array}{l}
n \\
k
\end{array}\right)_{q} x^{k} y^{n-k}, \quad 1 \leq k \leq n .
$$

where ()$_{q}$ is the $q$-binomial coefficient $([8])$ and the Wick product representation holds, that is,

$$
h_{k}\left(a_{f}, a_{f}^{\star}\right)=H_{k}^{q}\left(a_{f}+a_{f}^{\star}\right)
$$

where $H_{k}^{q}$ is the $k$-th $q$-Hermite polynomial and $a_{f}, a_{f}^{\star}$ are the annihilation and creation operators satisfying the $q$-commutation relation $a_{f} a_{f}^{\star}-q a_{f}^{\star} a_{f}=\mathbf{1}$ for a unit norm function $f$ in an infinite dimensional Hilbert space $([8])$.

Acknowledgments. Both authors extend special thanks to Prof. Friedrich Goetze. The second author would like to thank the organizing committee of the workshop for their hospitality.

Marek Bożejko was partially supported by Polish Ministry of Science and Higher Education grant no. N N201 3644 36, by the European Commission Marie Curie Host Fellowship for the Transfer of Knowledge "Harmonic Analysis, Nonlinear Analysis and Probability", MTKD-CT-2004-013389, by 7010 POLONIUM project "Non-Commutative Harmonic Analysis with Applications to Operator Spaces, Operator Algebras and Probability" and by joint PAN-JSPS project: "Noncommutative harmonic analysis on discrete structures with applications to quantum probability". Nizar Demni was supported by CRC 701.

\section{References}

[1] W. Al-Salam and T. S. Chihara, Convolutions of orthogonal polynomials, SIAM J. Funct. Anal. 7 (1976), 16-28. 
[2] N. Asai, I. Kubo and H. H. Kuo, Multiplicative renormalization and generating function I, Taiwan. J. Math. 7 (2003), 89-101.

[3] N. Asai, I. Kubo and H. H. Kuo, Generating functions of orthogonal polynomials and Szegö-Jacobi parameters, Probab. Math. Stat. 23 (2003), 273-291.

[4] M. Anshelevich, Free martingale polynomials, J. Funct. Anal. 201 (2003), 228-261.

[5] M. Anshelevich, Appell polynomials and their relatives, Int. Math. Res. Not. 65 (2004), 3469-3531.

[6] F. Benaych-Georges, Taylor expansions of R-transforms: application to supports and moments, Indiana Univ. Math. J. 55 (2006), 465-481.

[7] P. Biane, Processes with free increments, Math. Z. 227 (1998), 143-174.

[8] M. Bożejko, B. Kümmerer and R. Speicher, q-Gaussian processes: non-commutative and classical aspects, Comm. Math. Phys. 185 (1997), 129-154.

[9] M Bożejko and W. Bryc, On a class of free Lévy laws related to a regression problem, J. Funct. Anal. 236 (2006), 59-77.

[10] M. Bożejko and N. Demni, Generating functions of Cauchy-Stieltjes type for orthogonal polynomials, Infinite Dimen. Anal. Quantum Probab. Rel. Top. 12 (2009), 91-98.

[11] W. Bryc and M. E. H. Ismail, Approximation operators, q-exponential and free exponential families, Available on arXiv.

[12] W. Bryc and M. E. H. Ismail, Cauchy-Stieltjes kernel families, available on arXiv.

[13] M. Capitaine and M. Casalis, Asymptotic freeness by generalized moments for Gaussian and Wishart matrices, Application to beta random matrices, Indiana Univ. Math. J. 53 (2004), 397-431.

[14] T. S. Chihara, An Introduction to Orthogonal Polynomials, Gordon and Breach Science Publishers, 1978.

[15] B. Collins, Product of random projections, Jacobi ensembles and universality problems arising from free probability, P. T. R. F. 133 (2005), 315-344.

[16] N. Demni, Free Jacobi process, J. Theor. Probab. 21 (2008), 118-143.

[17] M. E. H. Ismail, Classical and Quantum orthogonal polynomials in one variable, Cambridge University Press, 2005.

[18] I. Kubo, Generating functions of exponential-type for orthogonal polynomials, Inf. Dimens. Anal, Quantum Probab. Related Topics 7 (2004), 155-159.

[19] I. Kubo, H. H. Kuo and S. Namli, The characterization of a class of probability measures by multiplicative renormalization, Communications on Stochastic Analysis 1 (2007), 455-472.

[20] R. G. Laha and E. Lukacs, On a problem connected with quadratic regression, Biometrika 47 (1960), 335-343.

[21] H. O. Lancaster, Joint probability distributions in the Meixner classes, J. Roy. Stat. Soc. Series B. 37 (1975), 434-443.

[22] J. Meixner, Orthogonale polynomsysteme mit einer besonderern der erzeugenden funktion, J. London. Math. Soc. 9 (1934), 6-13.

[23] C. N. Morris, Natural exponential families with quadratic variance function, Ann. Stat. 10 (1982), 65-80.

[24] W. Schoutens, Stochastic Processes and Orthogonal Polynomials, Lecture Notes in Statistics. Springer, 2000.

[25] I. M. Sheffer, Some properties of polynomial sets of type zero, Duke Math. J. 5 (1939), 590-622. 